\title{
Experiencia del uso de una app para evaluación en el aula
}

\author{
José Alfredo Núñez Toledo
}

\begin{abstract}
Resumen
El desarrollo de las Tecnologías de la Información y Comunicación (Tic) aplicadas en la educación ha permitido a los docentes disponer de una amplia gama de herramientas para innovar en el proceso de enseñanza-aprendizaje; en el cual, la evaluación juega un papel de suma importancia. Este trabajo describe la experiencia resultante del uso de la herramienta de respuesta de audiencia Plickers, durante la evaluación formativa de un grupo de estudiantes del Colegio de Ciencias y Humanidades (ccH), plantel Azcapotzalco, institución educativa pública mexicana de nivel medio superior, con el propósito de conocer su efecto en la evaluación. Dicha herramienta permitió vincular un sitio web, un dispositivo móvil y un paquete de marcadores Quick Response (QR) para realizar una evaluación formativa. En esa actividad, participaron 25 estudiantes de primer semestre de la asignatura de Taller de Cómputo. Los resultados mostraron que en el aula se generó un ambiente divertido que facilitó la interacción, participación, actitud positiva y competitividad entre los estudiantes. Además, esta herramienta ayudó al docente en la organización y control de las tareas de calificación, así como en el análisis de los resultados. Debido a lo anterior, y de acuerdo con los efectos observados al llevar a cabo dicha actividad, podría sugerirse a otros docentes la implementación de este tipo de herramientas para obtener un proceso de evaluación innovador.
\end{abstract}

Palabras clave: evaluación formativa, herramientas de respuesta de audiencia, Plickers, TIc.

\section{EFFECT OF USING AN AUDIENCE RESPONSE TOOL IN A FORMATIVE ASSESSMENT}

\begin{abstract}
The development of the Information and Communication Technologies (ICT) applied into education has allowed teachers a wide range of tools to innovate the teaching-learning process, in which the assessment plays a very important role. This work describes the resulting experience of using Plickers an audience response tool, during the formative assessment of a student group from Colegio de Ciencias y Humanidades ( $\mathrm{CCH}$ ), campus Azcapotzalco, a higher education, Mexican, public, educative institution, with the purpose of knowing its effect in the assessment. Such a tool allowed to bring together a web site, a mobile device and a Quick Response markers set (QR), so that a formative assessment can take place. 25 students, in first semester, from the Compute Workshop, participated in the activity. Results showed that a fun environment was generated in the classroom, which facilitated interaction, participation, positive attitude and competitiveness among students. Additionally, this tool helped the teacher in organizing and controlling grading tasks, as well as analyzing outcomes. Consequently, and according to the observed effects along the activity, Plickers could be suggested to other teachers, in order to implement this sort of tools as an innovative assessment process.
\end{abstract}

Keywords: formative assessment, audience response tool, Plickers, ICT.

Recepción: 15/11/2019. Aceptación: 28/07/2020. Dol: http://doi.org/10.22201/cuaieed.16076079e.2020.21.5.12 
Licenciado en informática y Maestro en Administración de Organizaciones por la Universidad Nacional Autónoma de México (UNAM), cuenta con diversos diplomados en Pedagogía y Tecnologías de la Información y Comunicación (Tic) para la enseñanza, Habilidades Directivas, Gestión empresarial, Administración de proyectos TIc e Investigación Educativa; además ha impartido diversos cursos y talleres sobre tecnología educativa para profesores y estudiantes del Colegio de Ciencias y Humanidades (ccH). Ha colaborado en la asesoría de alumnos en el club de robótica del plantel Azcapotzalco y se ha desempeñado como coordinador en el Programa Institucional de Asesoría de dicho plantel. Actualmente colabora en la Secretaría Estudiantil de la Dirección General de ccH.

\section{Introducción}

Los contextos educativos hoy en día representan grandes retos para la comunidad docente, particularmente en lo relativo a la evaluación formativa, considerada elemento fundamental del proceso de enseñanza-aprendizaje. Ésta tiene como uno de sus propósitos el conocimiento de la consecución de los aprendizajes por parte del alumnado.

Esos propósitos fueron establecidos en el programa de estudio correspondiente. Es por ello que el docente debe buscar nuevas formas que le permitan llevar a cabo la evaluación en condiciones favorables, lo cual podría conseguirse con la implementación de herramientas tecnológicas. Tal es el caso de aquéllas que se diseñan para recopilar información de un grupo de personas, conocidas como herramientas de respuesta de audiencia, y que, de acuerdo con Fuertes y Grimaldo (2016), permiten introducir procesos de participación activa en el aula de manera sencilla y con un costo de implementación mínimo, dado que muchos estudiantes acuden a las clases con algún tipo de dispositivo móvil. En este trabajo, se describe la experiencia resultante del uso de la herramienta de respuesta de audiencia Plickers durante la evaluación formativa de un grupo de estudiantes del Colegio de Ciencias y Humanidades (ccH), plantel Azcapotzalco, institución educativa pública, mexicana, de nivel medio superior, con la finalidad de conocer su efecto. 


\section{Desarrollo}

El ccH, es uno de los tres subsistemas de bachillerato con que cuenta la Universidad Nacional Autónoma de México (UnAm). Fue creado el 26 de enero de 1971, durante el rectorado del Dr. Pablo González Casanova, para atender la creciente demanda educativa de nivel medio superior en la zona metropolitana de la Ciudad de México, así como para impulsar la transformación académica de la propia Universidad con una nueva perspectiva curricular y nuevos métodos de enseñanza (Escuela Nacional Colegio de Ciencias y Humanidades [ENCCH], 2018).

El Colegio fundamenta su quehacer en la teoría constructivista del aprendizaje, que ubica al alumno en el centro del acto educativo y lo concibe como una persona capaz de transformar su medio y a sí mismo, que además es el responsable último de su propio aprendizaje. Así, el cch convierte la educación en un acto vivo y dinámico, en donde el docente funge como facilitador y guía del alumno durante todo el proceso de construcción de su conocimiento. El CCH define tres principios filosóficos que le caracterizan: aprender a aprender, aprender a hacer y aprender a ser (ENCCH, 2015).

En las etapas del proceso de enseñanza-aprendizaje, la evaluación tiene un papel fundamental, ya que permite obtener información sobre el grado de efectividad de las actividades realizadas conjuntamente entre docente y alumnado en el aula y fuera de ella; las cuales pretenden coadyuvar en la formación de los estudiantes, al adquirir nuevos conocimientos y permitirles su aplicación en la vida cotidiana.

Para los fines del presente trabajo, se entenderá por evaluación educativa a:

una actividad sistemática integrada dentro del proceso educativo, cuya finalidad es la optimización del mismo. Tiene por objeto proporcionar la máxima información para mejorar este proceso, reajustando los objetivos, revisando críticamente planes, programas, métodos y recursos, facilitando la máxima ayuda y orientación a los alumnos. Asimismo, permite elevar la calidad del aprendizaje y aumentar el rendimiento de los alumnos (Rosales, 2014, p.2)

Cabe señalar que, existen diferentes tipos de evaluación educativa: la diagnóstica, sumativa y formativa. Es la evaluación formativa a la que se hace referencia en este documento, y es aquella

que se realiza durante el desarrollo del proceso de enseñanza-aprendizaje para localizar las deficiencias cuando aún se está en posibilidad de remediarlas, esto es, introducir sobre la marcha rectificaciones a que hubiere lugar en el proyecto educativo y tomar las decisiones pertinentes, adecuadas para optimizar el proceso de logro del éxito por el alumno (Rosales, 2014, p.3).

En seguida, el autor de este trabajo sugiere que algunos de los propósitos principales de la evaluación formativa son:

a) Informar tanto al alumnado como al docente acerca del progreso alcanzado por el primero. 
b) Identificar oportunidades de mejora, tanto para el estudiante como para el docente, en el proceso de enseñanza-aprendizaje y actuar en consecuencia.

c) Identificar comportamientos y actitudes del alumnado durante el desarrollo del proceso de enseñanza-aprendizaje.

d) Detonar la motivación del alumnado para participar activamente, al obtener progreso en el aprendizaje.

Para llevar a cabo una evaluación formativa, el docente puede utilizar alguna de las distintas herramientas disponibles, que le faciliten obtener información sobre el progreso del alumnado en el logro de los aprendizajes. Entre ellas se pueden mencionar: cuestionarios, autoevaluación, lista de cotejo, mapas conceptuales, debates, ensayos, entrevista, etcétera, ya sea en su formato tradicional en papel y tinta o digital, echando mano de las Tecnologías de la Información y Comunicación (Tic).

Por ejemplo, se puede usar una herramienta de respuesta de audiencia (HRA), que consiste en un conjunto de recursos tecnológicos como teléfonos inteligentes (smartphones), tabletas, dispositivos móviles, sitios web, pizarrones electrónicos, entre otros, interconectados a través de una red de datos que generalmente es internet. Una HRA, permite recopilar información en tiempo real sobre las opiniones o elecciones que, con relación a un asunto o tema particular, manifiesta un grupo de personas localizado, ya sea en un salón de clase, auditorio o cualquier otro lugar. Dicha información es procesada por el sistema y los resultados obtenidos son almacenados en un repositorio que puede ser accedido posteriormente para su análisis.

\section{La experiencia}

La experiencia se desarrolló a través de una actividad didáctica denominada "Qué tanto te enRedaste", cuyo objetivo era evaluar los aprendizajes logrados por 25 estudiantes al finalizar una unidad temática del programa de estudios de la asignatura de Taller de Cómputo. Se incluía la realización de una evaluación formativa; para ello se usó la herramienta de respuesta de audiencia Plickers, conformada por una app, un sitio web y un conjunto de tarjetas escaneables, impresas con códigos de respuesta rápida O QR; además, se requirió de un proyector de video, una computadora de escritorio o laptop con acceso a internet, cuenta de usuario en el sitio web Plickers, un dispositivo móvil (smartphone) con sistema operativo Android, cámara y acceso a internet.

A continuación, se describen las fases de desarrollo de la experiencia.

\section{Fase de preparación}

El docente, de manera previa al inicio de la actividad, elaboró un banco de reactivos, los cuales alimentaron la HRA. Accedió al sitio web de Plickers para generar una cuenta de usuario y creó una "clase nueva", en la cual ingresó los nombres del alumnado que participaría en dicha actividad (ver imagen 1). 
Imagen 1. Clase Taller de Cómputo, creada en Plickers, con nombres del alumnado. Fuente: elaboración propia.

Imagen 2. Ejemplo de tarjeta QR. Fuente: elaboración propia.

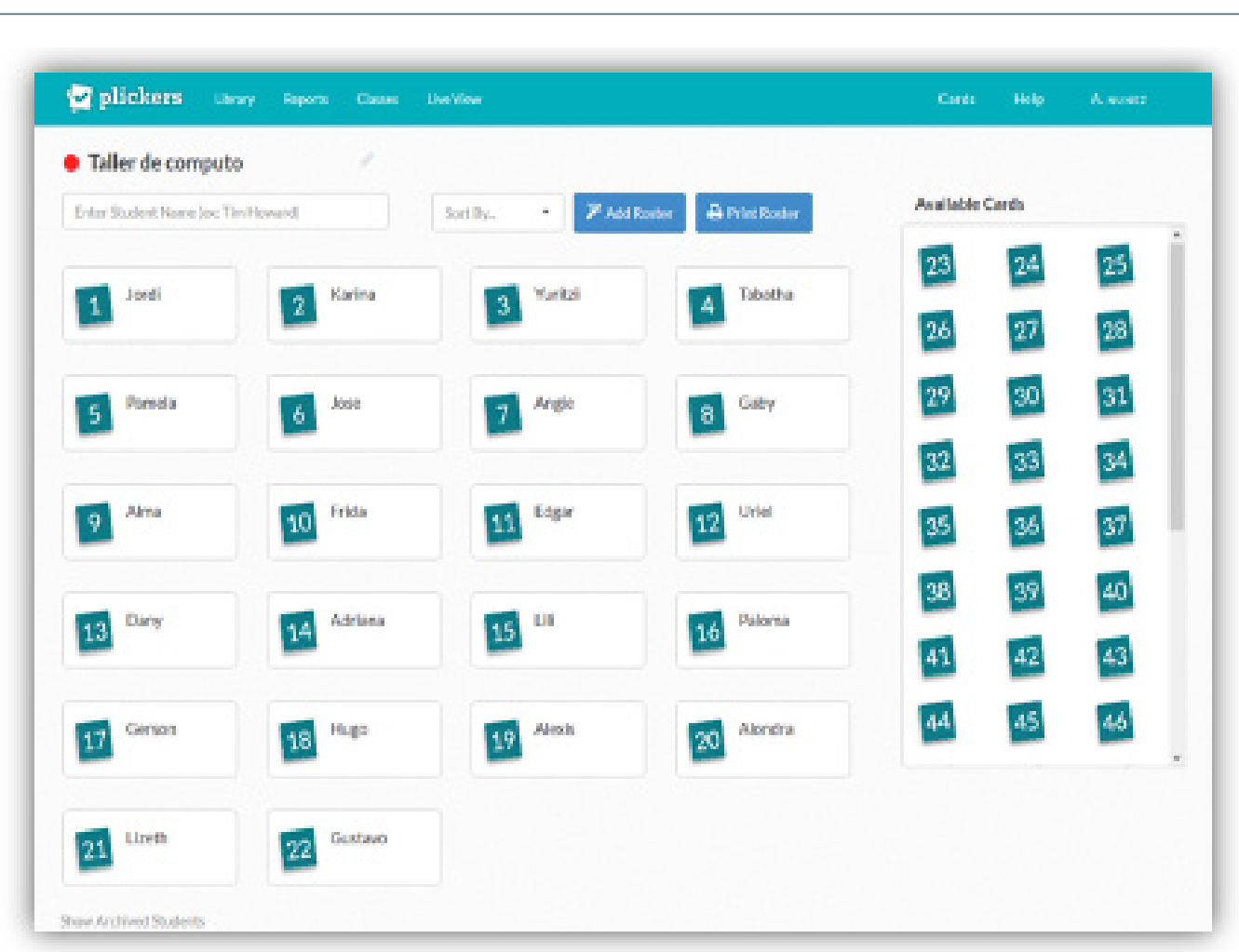

También, desde el sitio web Plickers se imprimieron las tarjetas QR, que identifican al estudiante por medio de un número. Éstas tienen cuatro posiciones que corresponden a las letras A, B, C y D, que representan las posibles opciones de respuesta a las preguntas (ver imagen 2).

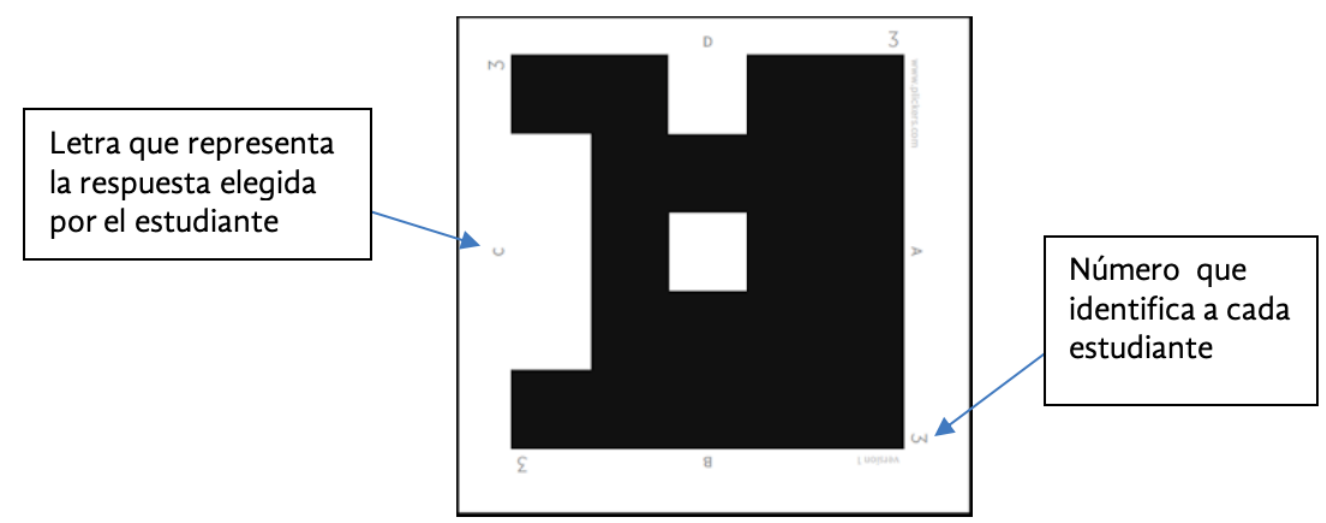

Usando la opción "Library" del mismo sitio web, se creó una librería y se añadieron en ella las preguntas seleccionadas del banco de reactivos que conformarían el instrumento de evaluación. En este caso, un cuestionario que se aplicaría a los estudiantes. Finalmente, se descargó e instaló la app de Plickers en un smartphone con sistema operativo Android, a través de la tienda de software en línea Google Play Store. 


\section{Fase de inicio}

En el salón de clase, el docente inició la actividad "Qué tanto te enRedaste" por medio de la siguiente pregunta detonadora: ¿se imaginan contestar un cuestionario usando una tarjeta, recolectar las respuestas con un celular y ver los resultados al instante en el pizarrón?, a continuación, explicó con detalle al alumnado en qué consistiría la actividad, dio las instrucciones necesarias para llevarla a cabo y repartió las tarjetas QR.

\section{Fase de ejecución}

El docente se conectó al sitio web y habilitó la opción "Live View". Utilizando el smartphone con la app Plickers instalada, comenzó a mostrar en el pizarrón, por medio del proyector de video, la primera pregunta del cuestionario. De esta forma, el alumnado pudo leer la pregunta.

Después de presentar la pregunta y cerciorarse de que había sido leída por el alumnado, el docente solicitó levantar sus tarjetas QR para indicar con su posición la respuesta que consideraban correcta; posteriormente, utilizando la cámara del smartphone desde la app Plickers, el docente procedió a recolectar o escanear las respuestas del alumnado, mismas que quedaron registradas en tiempo real en el sitio web Plickers (ver imagen 3).

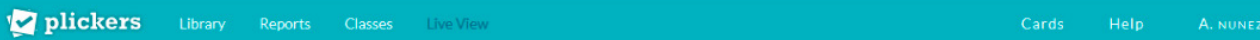

Es un conjunto de diferentes tipos de redes conectadas entre si alrededor de todo el mundo, capaz de facilitar el intercambio de información y uso compartido de recursos.
A: Redes de datos
B: Redes inalámbricas
C: Internet
D: Ninguna de las anteriores

Image 3. Vista de una de las preguntas proyectadas en el pizarrón y cómo se marcan al ser contestadas. Fuente: elaboración propia.
Tr

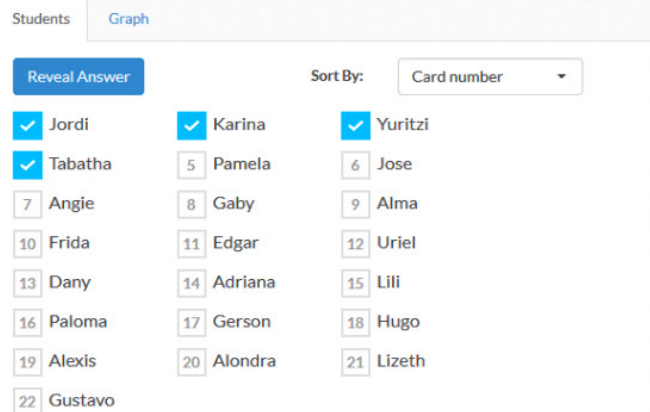

Al finalizar la recolección de respuestas, el docente pudo ver en la pantalla del smartphone quiénes habían contestado bien o mal, pero el alumnado sólo sabría los resultados hasta que éste decidiera mostrar las respuestas de cada estudiante en el pizarrón. Después de preguntarles si estaban seguros de sus respuestas, el docente, dando un clic, hizo que la herramienta mostrara, por medio de los colores verde (correcto) y rojo (incorrecto), los resultados obtenidos. Al verlos, varios alumnos y alumnas saltaron de alegría al descubrir que habían contestado correctamente, mientras que otros se dieron cuenta de que no lo habían hecho y permanecieron quietos. 
Imagen 4. Izquierda. Alumnado respondiendo mediante tarjetas QR. Derecha. Docente recolectando la información. Fuente: elaboración propia.

Video 1. Video de la dinámica (Nunez, 2016a).
Este proceso de pregunta-respuesta-escaneo-resultado se realizó de forma iterativa hasta completar las preguntas contenidas en el cuestionario, generando un ambiente dinámico, participativo, competitivo y muy divertido (ver imagen 4). Para conocer cómo se llevó a cabo la dinámica de dicho proceso en el aula, se puede observar la videograbación disponible (ver video 1).
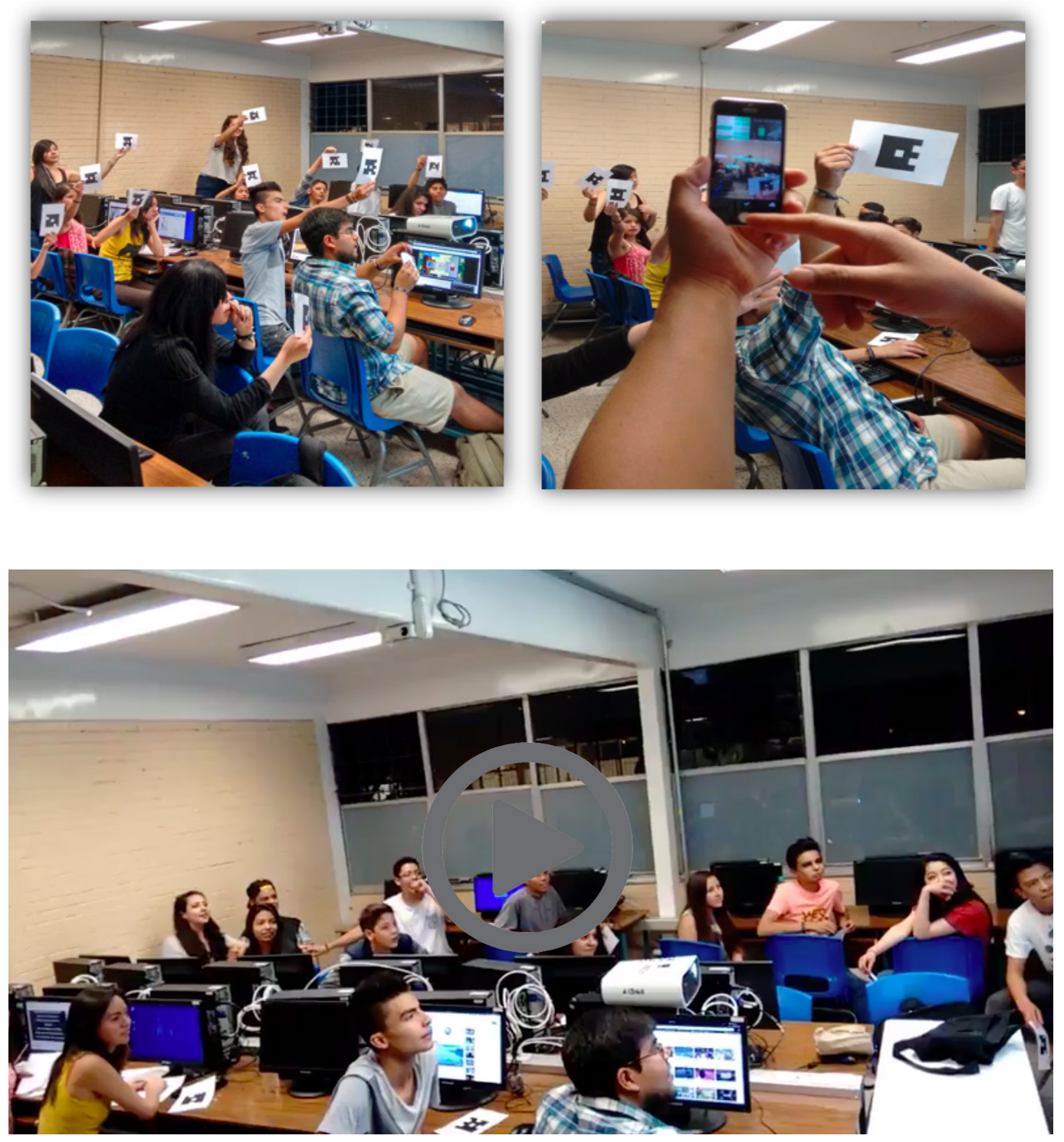

\section{Fase de cierre}

Por medio de la opción "Reports", el docente pudo conocer los resultados obtenidos en el cuestionario por los estudiantes de forma rápida y fácil, tanto de forma individual como grupal. En conjunto, el porcentaje promedio de respuestas correctas fue de $83 \%$. Con esta información, el docente puede realizar un análisis de resultados con la finalidad de identificar aquellos aspectos 
Imagen 5. Resultados porcentuales obtenidos sobre actitud y satisfacción de los estudiantes al usar Plickers. Fuente: Elaboración propia. en los que el alumnado presentó dificultades y, en consecuencia, trabajar en la elaboración de un plan de mejora que coadyuve a solventar las deficiencias encontradas en el logro de los aprendizajes.

Posteriormente, el profesor solicitó a los estudiantes valorar de forma individual las siguientes afirmaciones para conocer su actitud y satisfacción después de utilizar Plickers como herramienta de evaluación formativa:

1. Es una forma innovadora y divertida de hacer una evaluación.

2. Me gustó esta forma de hacer evaluaciones.

3. Preferiría ser evaluado utilizando herramientas como ésta.

Para medir los resultados obtenidos de dicha valoración de afirmaciones, se utilizó una escala de Likert, con cinco niveles de respuesta: totalmente en desacuerdo, en desacuerdo, ni de acuerdo ni en desacuerdo, de acuerdo y totalmente de acuerdo. Se encontró que de una población de 25 estudiantes que respondieron, con respecto a la pregunta 1 (P1), 92\% estuvo totalmente de acuerdo y $8 \%$ de acuerdo; para la pregunta 2 (P2), 100\% estuvo totalmente de acuerdo; finalmente, en relación con la pregunta 3 (P3), 88\% estuvo totalmente de acuerdo, $8 \%$ de acuerdo y $4 \%$ totalmente en desacuerdo (ver imagen 5).

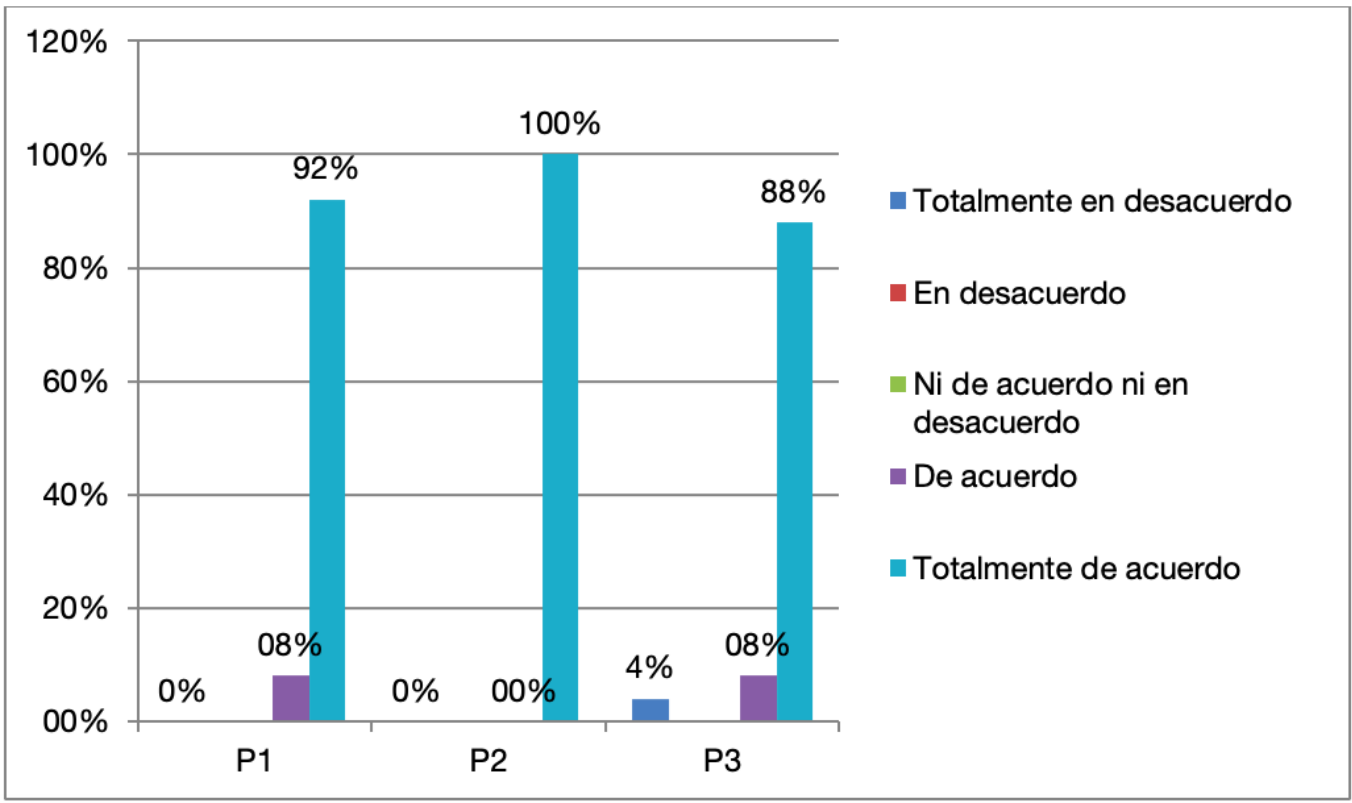

Para finalizar, el profesor en plenaria, invitó a los alumnos a compartir su experiencia con el grupo después de realizar la evaluación formativa con Plickers. Al término de algunas participaciones, destacó ciertos puntos importantes de lo expuesto, agradeció la colaboración de los estudiantes y dio por concluida la actividad. 


\section{Conclusiones}

La utilización de la herramienta Plickers permitió la participación activa del alumnado, que se mostró motivado e interesado por responder a las distintas preguntas. La interacción entre docente y alumnado se dio de forma natural, el ambiente era muy divertido y competitivo, hubo momentos en que alumnas y alumnos discutían entre ellos para decidir cuál era la respuesta correcta, algunos festejaban sus aciertos saltando en sus lugares y dando un fuerte grito, chocando las palmas con su compañeros o dándose un abrazo. En cambio, aquéllos que no acertaban se mantenían quietos y callados, pero dispuestos a lograrlo en la siguiente oportunidad. La actitud del alumnado fue muy positiva, lo cual puede constatarse en los siguientes videos que contienen sus testimonios (ver videos 2 y 3 ).

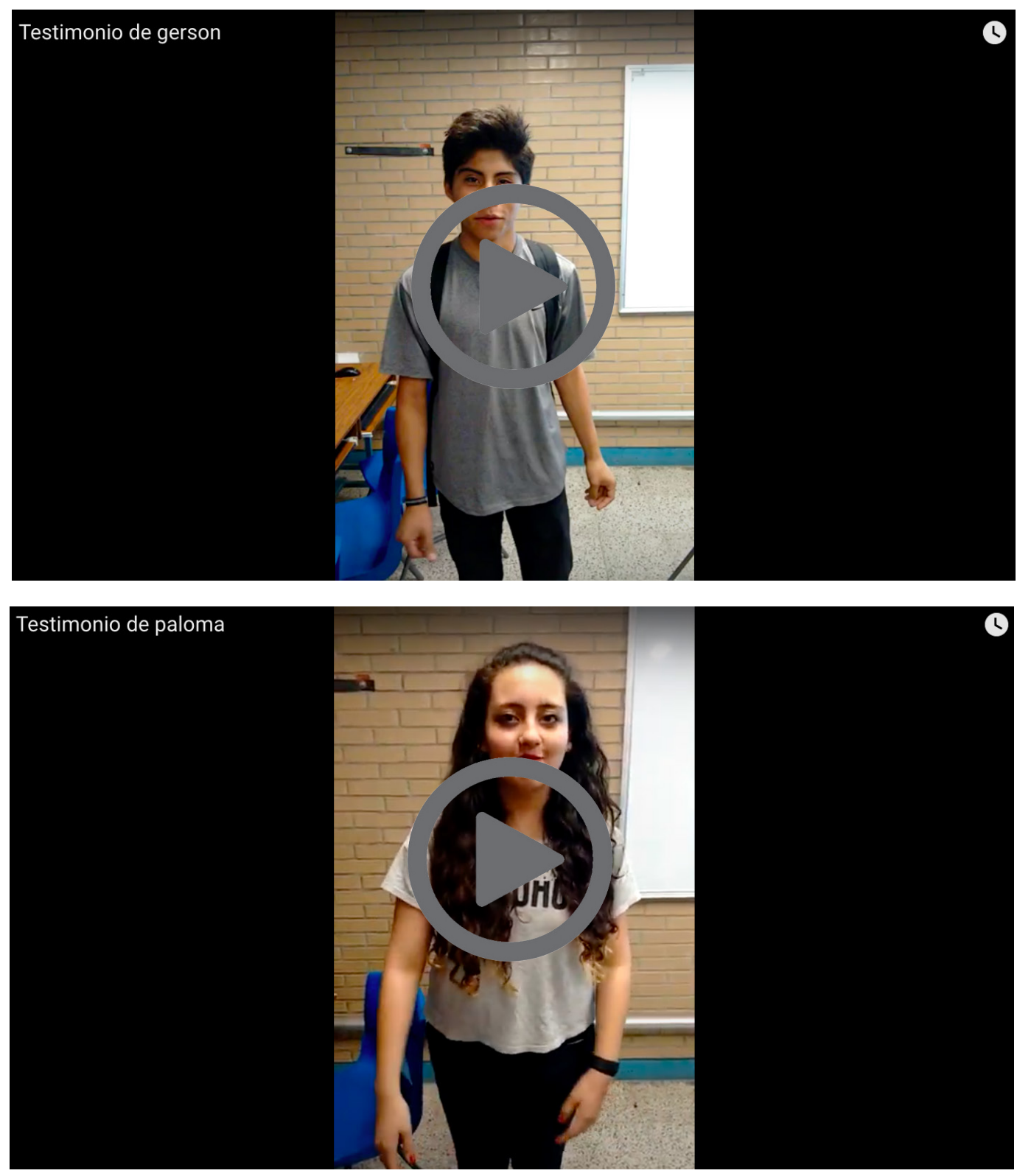


Los estudiantes que participaron en la actividad evaluativa con el uso de la herramienta Plickers manifestaron estar satisfechos y algunos plantearon la necesidad de que los profesores y profesoras "modifiquen su manera tradicional de enseñar", lo que sugiere el desarrollo de estrategias didácticas innovadoras apoyadas en TIC.

Ante tal situación, el docente debe estar dispuesto a adoptar una postura crítica sobre su propuesta educativa, escuchar lo que el estudiante opina al respecto y reconocerlo como elemento fundamental y central del proceso de enseñanza-aprendizaje, así como comprender de mejor manera sus motivaciones y la realidad actual en la que están inmersos y, además, ver al cambio como una oportunidad para mejorar. La innovación educativa a través del uso de tı se convierte en una gran aliada para enfrentar los nuevos retos que su quehacer docente le presenta.

La decisión de utilizar Plickers responde a la necesidad de explorar nuevas formas de abordar los retos que presenta la evaluación educativa, la cual, derivó en una experiencia que permitió, entre otras cosas, establecer una relación en la que tanto docente como estudiante ganaban, pues se pudo desarrollar una actividad didáctica que involucró la evaluación formativa de los estudiantes y que fue bien aceptada por ellos, y les brindó información valiosa sobre la consecución de los aprendizajes establecidos en el programa de estudio. Además, se logró su participación activa en un ambiente divertido, que incrementó la motivación por aprender de forma lúdica y diferente; asimismo, se acercó a los estudiantes al conocimiento de nuevas posibilidades tecnológicas y conceptuales, que ellos mismos reconocieron como importantes y útiles para su desarrollo educativo. Por último, la actitud mostrada por alumnas y alumnos, durante y después de la actividad, fue muy positiva. Incluso, en algunos casos, se manifestó el agradecimiento hacia el docente, por preocuparse en buscar nuevas formas para facilitarles el aprendizaje, que no fueran aburridas ni tradicionales.

Cabe señalar que, este trabajo, no fue concebido como una investigación rigurosa, sino como el desarrollo de una idea considerada innovadora para explorar alternativas y que afortunadamente dio como resultado una buena experiencia de aprendizaje.

\section{Referencias}

* Alonso Martín, M. del C. (2010, junio). Variables del aprendizaje significativo para el desarrollo de las competencias básicas. http://www.aprendizajesignificativo. es/mats/Variables\%20del\%20aprendizaje\%20significativo\%20para\%20el\%20 desarrollo\%20de\%20las\%20competencias\%20basicas.pdf

* Escuela Nacional Colegio de Ciencias y Humanidades (ENCCH). (2018). Historia del Colegio de Ciencias y Humanidades. https://www.cch.unam.mx/historia 
* Escuela Nacional Colegio de Ciencias y Humanidades (ENCCH). (2015). Modelo Educativo del Colegio de Ciencias y Humanidades. http://www.cch.unam.mx/ sites/default/files/MODELO\%20EDUCATIVO\%20DEL\%20COLEGIO\%20DE\%20 CIENCIAS\%20Y\%2OHUMANIDADES.pdf

* Fuertes Seder, A. y Grimaldo Moreno, F. (2016). Motivación, participación y aprendizaje del alumnado mediante el uso de herramientas de respuesta de audiencia. En J. F. Durán Medina e I. Durán Valero (dirs.), tıc actualizadas para una nueva docencia universitaria (233-245). McGraw-Hill Interamericana de España. https://dialnet.unirioja.es/servlet/articulo?codigo=6838392

* Nunez, A. (2016a, 6 de mayo). EvaluaciónGrupo267A_CCHAzcapotzalco [video]. YouTube. https://youtu.be/B8Ue8lleusw

Nunez, A. (2016b, 6 de mayo). Testimonio de gerson [video]. YouTube. https://youtu. be/Y4gqMRvKY5g

* Nunez, A. (2016c, 6 de mayo). Testimonio de paloma [video]. YouTube. https:// youtu.be/WT4W1JSmP_E

- OCDE. (2005). Formative assesment: improving learning in secondary classrooms. https://www.oecd.org/edu/ceri/35661078.pdf

* Plickers. (2016). Welcome to Plickers. https://plickers.zendesk.com/hc/en-us

* Rosales, M. (2014). Proceso evaluativo: evaluación sumativa, evaluación formativa y Assesment su impacto en la educación actual. https://www.oei.es/historico/ congreso2014/memoriactei/662.pdf

\section{Cómo CITAR ESTE ARTículo}

* Núñez Toledo, José Alfredo. (2020, septiembre-octubre). Experiencia del uso de una app para evaluación en el aula. Revista Digital Universitaria (RDU), 21(5). DOI: http://doi.org/10.22201/cuaieed.16076079e.2020.21.5.12 